\title{
An approach to the metabolic degradation of diethylketone (DEK) by Streptococcus equisimilis: Effect of DEK on the growth, biodegradation kinetics and efficiency
}

\author{
Filomena Costa, Cristina Quintelas*, Teresa Tavares \\ IBB-Institute for Biotechnology and Bioengineering, Centre of Biological Engineering, University of Minho, Campus de Gualtar, 4710-057 Braga, Portugal
}

\section{A R T I C L E I N F O}

\section{Article history:}

Received 16 December 2013

Received in revised form 16 April 2014

Accepted 18 May 2014

Available online 14 June 2014

\section{Keywords:}

Biodegradation kinetics

Degradation

Diethylketone

Growth kinetics

Metabolic pathway

Streptococcus equisimilis

\begin{abstract}
A B S T R A C T
The degradation of diethylketone by Streptococcus equisimilis was evaluated. The toxicity of diethylketone was assessed evaluating the growth of the bacteria for the range of diethylketone concentration between 0 and $6.4 \mathrm{~g} \mathrm{~L}^{-1}$. The maximum specific growth rate achieved is $0.555 \mathrm{~h}^{-1}$ at $3.2 \mathrm{~g} \mathrm{~L}^{-1}$ of initial diethylketone concentration, followed by a slight decrease, suggesting that higher concentrations of diethylketone negatively affect the growth. The biodegradation efficiency (\%) obtained was approximately 95\%, for all the initial concentrations tested. The kinetic parameters were estimated using Monod, Powell, Haldane and Loung models. The experimental data is well fitted by the Loung and Haldane models, $R^{2}=0.98$ for both, as compared to Monod model $\left(R^{2}=0.79\right)$ and Powell model $\left(R^{2}=0.72\right)$. Once the previous intrinsic functions were established, a set of assays was also performed to evaluate the biodegradation of diethylketone using concentrated biomass for initial diethylketone concentrations ranging from 0.8 to $3.9 \mathrm{~g} \mathrm{~L}^{-1}$ in a bioreactor operating in batch mode with recirculation. These concentrated biomass assays aimed the optimization of operational conditions. The removal percentages obtained were approximately $100 \%$, for all the initial ketone concentrations tested and the biodegradation rate followed the pseudo-second order kinetics. The mechanism involved in the degradation of diethylketone by this microorganism is not completely understood, but an approach to the metabolic degradation pathway was established by the identification of the metabolites involved on the process.
\end{abstract}

(c) 2014 Elsevier B.V. All rights reserved.

\section{Introduction}

The increasing use of solvents in industrial activities and the more and more restrictive legislation on their discharge into the environment justify the need for new and cost- effective treatment of industrial wastewater. As almost all the solvents, diethylketone is dangerous to aquatic life in high concentrations. According to Tani and Hewitt (2009) the C4-C6 ketones are absorbed by plants leaves and can enter in the food chain of animals and humans with severe consequences. In this context, the identification of ketonedegrading microorganisms and the understanding of pre-requisites and of mechanistic pathways used to metabolize those compounds are imperative.

Biological treatments as biosorption, enzymatic degradation or others are, in general, less expensive than physicochemical

\footnotetext{
* Corresponding author. Tel.: +351 253604400; fax: +351 253604429

E-mail address: cquintelas@deb.uminho.pt (C. Quintelas).
}

methods for wastewater treatment (Das et al., 2014; Akar et al., 2013; Lin et al., 2013). In the last years some works have been published using biosorbents to solve the problem of solvent spreading. The removal of diethylketone was studied by Quintelas et al. (2013) who used a combination of microorganisms and clays and by Costa et al. (2012) who used Arthrobacter viscosus to biodegrade this solvent. In fact, the main difference between the present report and those last two works is the use of a different bacterium (Streptococcus equisimilis) as free bacteria (in suspension).

The development of a technology applicable to the treatment of aqueous solutions with low concentrations of solvents is the aim of this work. In particular, the detailed kinetic study of the biodegradation of diethylketone is one of the focuses of this research. The information collected from the experimental studies was used for the calculation of growth kinetic constants with different models - Monod (1949), Powell (1967), Haldane (Andrews, 1968) and Luong (1986). The kinetics of biodegradation was analyzed by zero order, pseudo-first order, pseudo-second order and threehalf order models. A new approach to the metabolic degradation 
pathway and to the mechanism involved in the degradation of DEK by this microorganism is presented and the identification of the metabolites involved on the removal process is also presented. These important findings constitute an upgrade of the state of the art concerning the application of biosystems to the rehabilitation of contaminated waterbodies and an important contribution to the ecological engineering.

\section{Materials and methods}

\subsection{Materials}

Aqueous diethylketone solutions were prepared by diluting diethylketone (Acros Organics) in distilled water. The microorganism used on the degradation assays was S. equisimilis (CECT 926) from the Spanish Type Culture Collection - University of Valencia. The growth media was the Brain Heart Infusion CM0225 (BHI) from Oxoid.

\subsection{Methods}

\subsubsection{Effect of diethylketone on the growth}

The media prepared contained $37 \mathrm{~g} \mathrm{~L}^{-1}$ of $\mathrm{BHI}$ in distilled water. The $\mathrm{pH}$ was adjusted to 7.4 . A reactor $(750 \mathrm{~mL})$ was filled with $500 \mathrm{~mL}$ of this medium and autoclaved. The reactor was then inoculated with a pure culture (initial biomass concentration circa $0.3 \mathrm{~g} \mathrm{~L}^{-1} ; 15 \mathrm{~mL}$ of inoculum) of $S$. equisimilis and a pre-determined amount of diethylketone was added $\left(0-6.4 \mathrm{~g} \mathrm{~L}^{-1}\right)$. The diethylketone concentrations were selected based on local typical values present in effluents containing solvents and based in previous studies (Costa et al., 2012) where concentrations up to $4.8 \mathrm{~g} \mathrm{~L}^{-1}$ were used. A control experiment without diethylketone was also performed. The reactor was equipped with a heating jacket to keep the temperature constant $\left(37^{\circ} \mathrm{C}\right)$. The agitation was implemented by medium recirculation and by a magnetic stirrer. At different time intervals, a sample was withdrawn and the optical density (OD) was measured at $620 \mathrm{~nm}$ (T60 UV-Visible Spectrophotometer, PG Instruments). The initial concentration of diethylketone was quantified using GC (Chrompack CP 9001) equipped with a flame ionization detector (FID) (Quintelas et al., 2011). All the assays were done in duplicate and the results presented are an average of both assays. The relative standard deviation and relative error of the experimental measurements were less than $2 \%$ and $5 \%$, respectively, for the assays in Sections 2.2.1 and 2.2.2.

The biodegradation efficiency (\%) was calculated accordingly to:

Biodegradation efficiency $(\%)=\frac{C_{0}-C}{C_{0}} \times 100$

$C_{0}$ and $C$ are the initial and the instantaneous concentrations of the ketone.

\subsubsection{Biodegradation}

S. equisimilis was grown for $24 \mathrm{~h}$ at $37^{\circ} \mathrm{C}$ in $500 \mathrm{~mL}$ of a culture medium with the composition described in Section 2.1 and then 3 sets of $150 \mathrm{~mL}$ of this inoculated medium were transferred to 3 new culture media $(1000 \mathrm{ml})$ and these cultures were grown for $48 \mathrm{~h}$. Then, the biomass was centrifuged on a Sigma $4 \mathrm{~K} 15$ centrifuge (RCF 7950)(20 min; $9000 \mathrm{rpm}$ ) and the biomass pellets were used to inoculate the reactors filled with a diethylketone solution $(500 \mathrm{~mL})$. The biomass concentration is, approximately, $6.0 \mathrm{~g} \mathrm{~L}^{-1}$. The diethylketone concentrations used in these studies were on the range $0.8-3.9 \mathrm{~g} \mathrm{~L}^{-1}$. Diethylketone was supplied as the only source of carbon and energy. The biomass concentration for this second set of assays was calculated using a calibration curve (optical density vs. biomass concentration) and the measurement of the dry weight.
At different time intervals, a sample was taken, centrifuged (MiniSpin, Eppendorf) at 13,400 rpm for $10 \mathrm{~min}$, and the diethylketone concentration was determined in the supernatant by GC. During the analysis three main metabolites appear in the chromatograms and they were identified by comparison of the retention time of known pure compounds. The assays were conducted in duplicate, during circa 7 days and the results are an average of both assays.

\subsubsection{Modelling of the growth and biodegradation kinetics}

In the present study, the linear and nonlinear growth kinetic models were fitted by linear and nonlinear least squares methods using MATLAB software. The models used were Monod, Powell, Haldane and Loung, for growth kinetics and zero order, pseudo-first, pseudo-second and three-half order equations, for biodegradation kinetics and were well described in Costa et al. (2012).

\section{Results and discussion}

In order to investigate the growth kinetics of S. equisimilis in the presence of the ketone, a number of growth experiments were performed in batch mode. The first set of experiments aimed to study the xenobiotic effect of diethylketone on the growth and to model this specific growth kinetics. The biomass was then concentrated and used in a second set of experiments for the analysis of the biodegradative skills of this bacterial species. These biodegradation assays led to the reduction of the diethylketone concentration and the kinetics of this process were also modelled.

\subsection{Effect of diethylketone on the bacteria growth}

The growth of $S$. equisimilis was analyzed in the presence and absence of diethylketone. The bacteria grew exponentially during $4 \mathrm{~h}$ in $\mathrm{BHI}$ medium and without diethylketone, with a specific growth rate of $0.43 \mathrm{~h}^{-1}$. In order to examine the toxic effects of diethylketone on the growth, cells of Streptococcus were incubated in media containing diethylketone (1.6, 3.2 and $\left.6.4 \mathrm{~g} \mathrm{~L}^{-1}\right)$. Fig. 1(a) shows phases lag and log of the growth curves.

Usually the specific growth rate increases with an increase in substrate (diethylketone) concentration until a maximum value is reached. In this work, for concentrations higher than $3.2 \mathrm{~g} \mathrm{~L}^{-1}$ the specific growth rate started to decrease, indicating substrate inhibition of growth. According to Hasan et al. (2012), this may be due to cell damage or disruption of membrane integrity at higher diethylketone concentrations. The results also show that the "acclimation time", lag phase, is longer for the highest concentrations. In terms of specific growth rate the difference is not so evident, as it decreases from 0.5546 to $0.4997 \mathrm{~h}^{-1}$, although the lag phase duration increases from 5 to $11 \mathrm{~h}$. These results also confirm the substrate inhibition of growth.

The biodegradation efficiency (\%) (Eq. (1)) was calculated and a value of 95\% was obtained for all the assays. In comparison with previous works (Costa et al., 2012) on the biodegradation of diethylketone with a different microorganism (A. viscosus), it is possible to conclude that $S$. equisimilis has a better performance. This microorganism is not so sensitive to the xenobiotic effect of diethylketone as it presents higher specific growth rates (ex: $0.5546 \mathrm{~h}^{-1}$ compared to $0.1737 \mathrm{~h}^{-1}$ for $A$. viscosus, with an initial diethylketone concentration of $3.2 \mathrm{~g} \mathrm{~L}^{-1}$ ) and this better growth allows better biodegradation efficiency, from $88 \%$ for $A$. viscosus, to 95\% for S. equisimilis. 

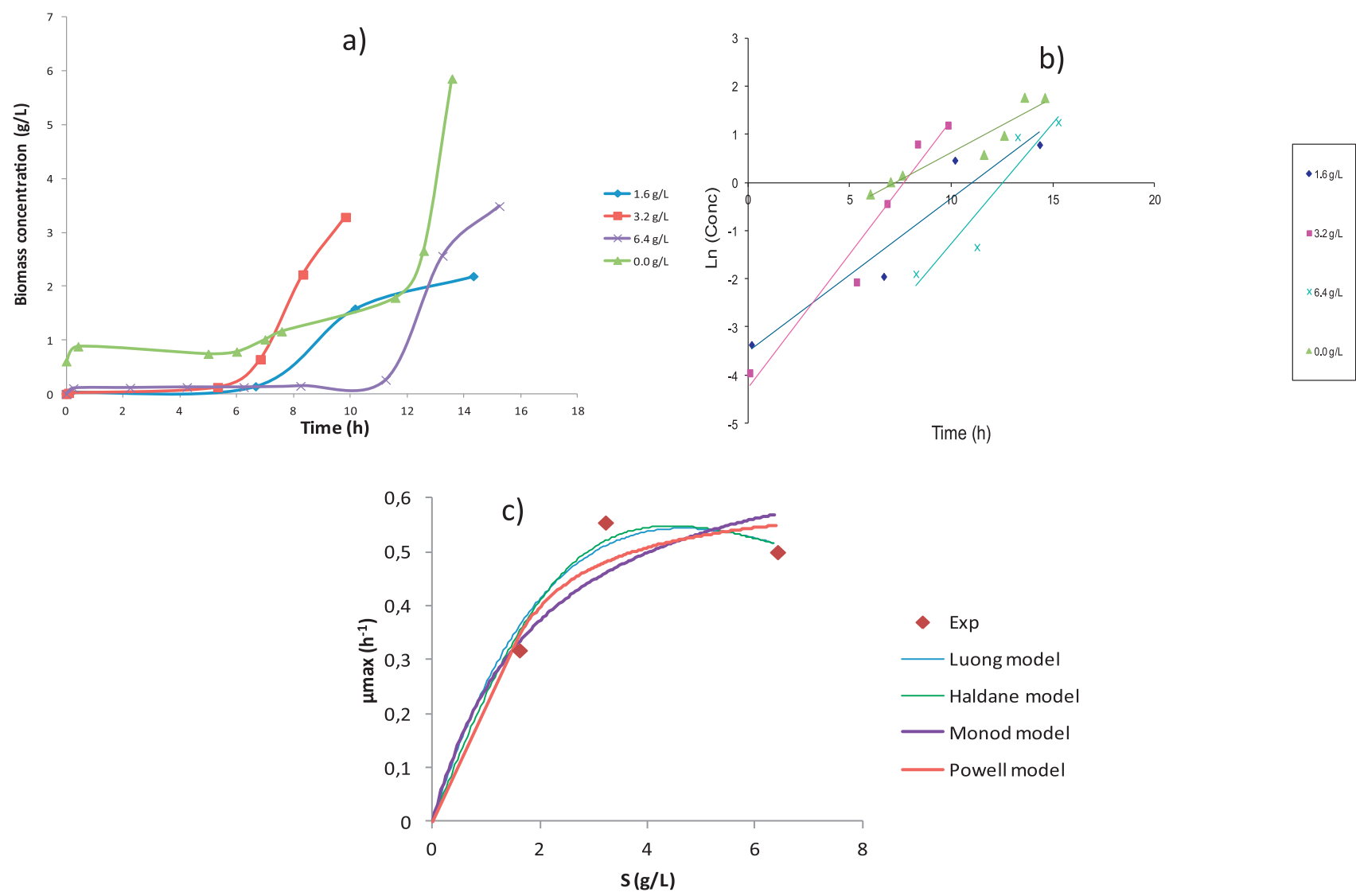

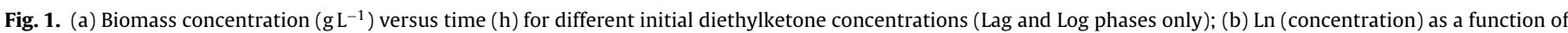
time for the determination of the specific growth rate; (c) comparison between the experimental results and those predicted by the Loung and Haldane models.

\subsection{Modelling of the growth kinetics of S. equisimilis in the presence of diethylketone}

An understanding of the growth kinetics is very important for the design of a process for the treatment of hazardous wastewater. To calculate the specific growth rates, the biomass concentration was measured at several time intervals. A plot of Ln (concentration) versus t gives a straight line where $\mu$ (specific growth rate) is the slope (Fig. 1(b)). The obtained values of specific growth rates are $0.4331,0.3177,0.5546$ and $0.4997 \mathrm{~h}^{-1}$, respectively for the initial diethylketone concentrations of 0 , $1.6,3.2$ and $6.4 \mathrm{~g} \mathrm{~L}^{-1}$. For the assays performed in the presence of diethylketone, the decrease verified on the specific growth rate for initial diethylketone concentrations higher than $3.2 \mathrm{~g} \mathrm{~L}^{-1}$ suggested inhibition by this ketone. Comparing again with the results obtained when using A. viscosus (Costa et al., 2012), it was verified that $S$. equisimilis is less sensitive to the xenobiotic effect because this was only detected for diethylketone concentrations higher than $3.2 \mathrm{~g} \mathrm{~L}^{-1}$ against $1.6 \mathrm{gL}^{-1}$, detected for $A$. viscosus.

Various theoretical models such as Monod, Powell, Haldane and Luong kinetic models were used in this study and the growth kinetic parameters obtained for the different growth models as well as the comparison between experimental data and Powell model predicted data are presented in Table 1.

For the Monod model the values of $\mu_{\mathrm{m}}$ and $K_{\mathrm{s}}$ were calculated from the plot of $1 / \mu$ vs. $1 / \mathrm{S}$. The values of $\mu_{\mathrm{m}}$ and $K_{\mathrm{s}}$ obtained were $0.7525 \mathrm{~h}^{-1}$ and $2.004 \mathrm{~g} \mathrm{~L}^{-1}$ respectively. The value of $K_{\mathrm{s}}$ obtained is much smaller than $3.2 \mathrm{~g} \mathrm{~L}^{-1}$ which confirms the inhibitory effect of diethylketone above this concentration. The obtained value of correlation coefficient $\left(R^{2}\right)$ was 0.79 indicating that this model does not fit properly the data.

Table 1

Growth models kinetic parameters, experimental and predicted values of specific the growth rate.

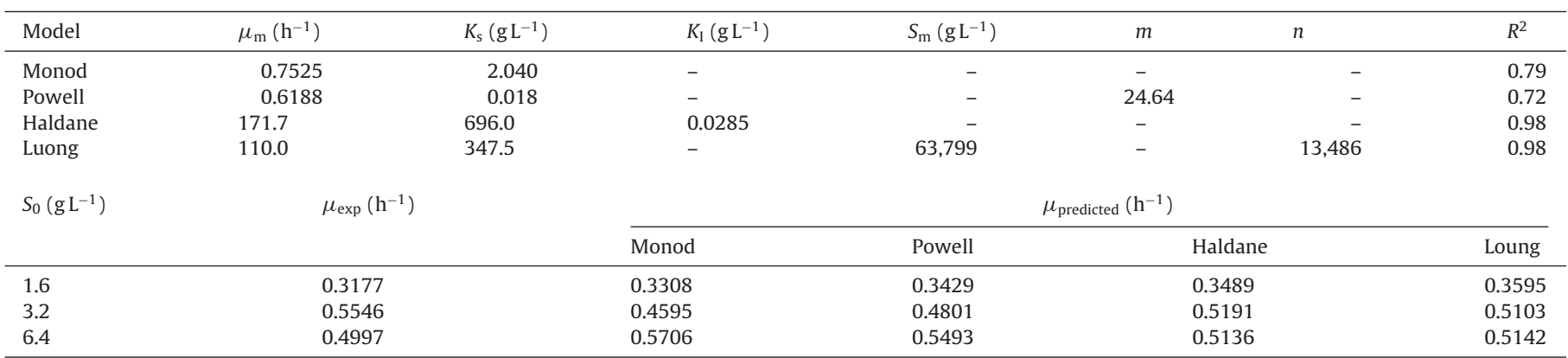


a)

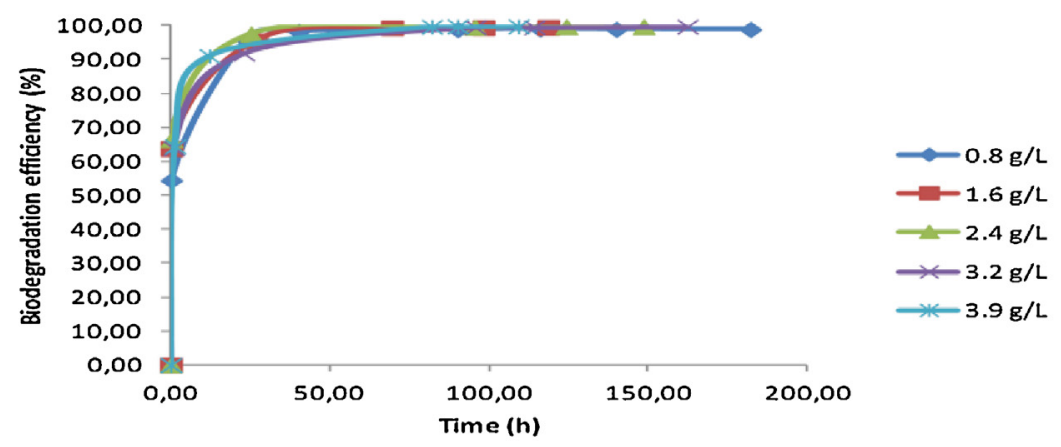

b)

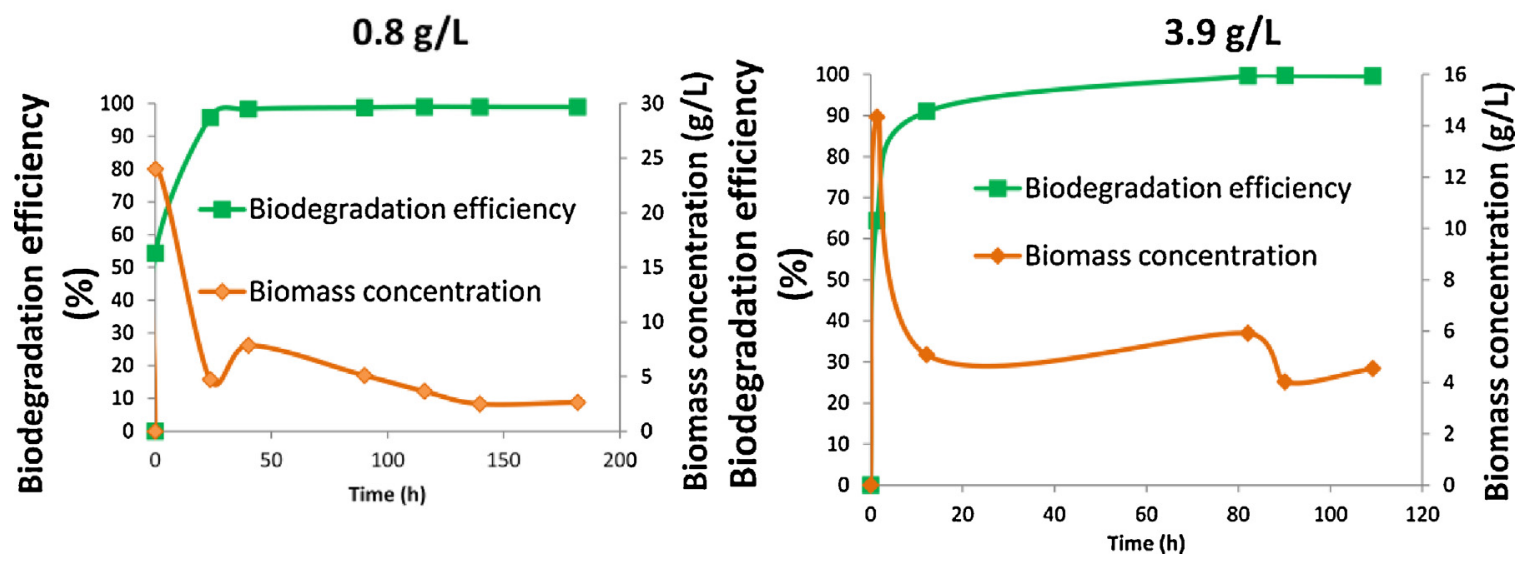

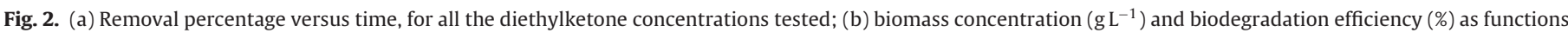
of time, for the lower and the higher diethylketone concentrations ( 0.8 and $\left.3.9 \mathrm{~g} \mathrm{~L}^{-1}\right)$;.

The parameters obtained for Powell model were $0.6188 \mathrm{~h}^{-1}$, $0.018 \mathrm{~g} \mathrm{~L}^{-1}$ and 24.64 , respectively for $\mu \mathrm{m}, K_{\mathrm{s}}$ and $\mathrm{m}$ (Table 1$)$. The obtained value of correlation coefficient $\left(R^{2}\right)$ was 0.72 which indicates that, as for Monod model, this model does not fit properly the data.

The bad fit obtained with these two models is not unexpected as these models do not consider the inhibition effect detected during the diethylketone biodegradation process. As for the Monod model, the value of $K_{\mathrm{S}}$ obtained is smaller than $3.2 \mathrm{~g} \mathrm{~L}^{-1}$, confirming the inhibition effect.

The parameters for Haldane and Loung models were calculated and are presented in Table 1 and the fit of both models to the experimental results and the predicted values of specific growth rate at different concentrations are listed in Table 1 and Fig. 1(c). The parameters calculated for Haldane model were $171.7 \mathrm{~h}^{-1}, 696 \mathrm{~g} \mathrm{~L}^{-1}$ and $0.0285 \mathrm{~g} \mathrm{~L}^{-1}$, respectively for $\mu \mathrm{m}, K_{\mathrm{S}}$ and $K_{\mathrm{I}}$ and for Loung model were $110.0 \mathrm{~h}^{-1}, 347.5 \mathrm{~g} \mathrm{~L}^{-1}, 63,799 \mathrm{~g} \mathrm{~L}^{-1}$ and 13,486 , respectively for $\mu \mathrm{m}, K_{\mathrm{s}}, S_{\mathrm{m}}$ and $n$. The value of correlation coefficient $\left(R^{2}=0.98\right)$ showed that the present data are well fitted by the Haldane and Loung models, as it may be seen in Fig. 1(c). In Table 1 it is evident that the $\mu_{\text {predicted }}$ is closer to the $\mu_{\text {exp }}$ for these two models than for Monod and Powell models, which proves that the inhibition models explain better the growth kinetics in the presence of diethylketone than the other models used.

\subsection{Biodegradation assays}

It is expected that high concentration of the biomass (small ratio solvent/biomass) may promote the biodegradation efficiency. The concentrated biomass was added to a diethylketone solution (only diethylketone diluted in water) to avoid any interference of other carbon sources.

Fig. 2(a) represents the biodegradation efficiency as a function of time, for all the diethylketone concentrations tested. The biodegradation is fast on the first hours, after which it slows down until $100 \%$ efficiency is reached. This initial fast removal is explained by the availability of the biomass and the need of the biomass to consume carbon. After this period of time, the biodegradation efficiency slows down due the saturation of the biomass. In Fig. 2(b), it is possible to analyze the relationship between the biomass concentration and the biodegradation efficiency, for the lowest and for the highest diethylketone concentrations used. An obvious conclusion is that the biomass grows slightly with the consumption of diethylketone and decreases as the diethylketone disappears. For the lowest solvent concentration the maximum of biomass concentration achieved is higher, $24 \mathrm{~g} \mathrm{~L}^{-1}$ against $14 \mathrm{~g} \mathrm{~L}^{-1}$ for the highest solvent concentration, probably due to the xenobiotic effect established for concentrations starting from $3.9 \mathrm{gL}^{-1}$ onwards. As the carbon source is being depleted, the biomass decreases, for all the concentrations tested.

The process of biodegradation is very complex and could be a result of several factors as enzymatic degradation, binding by functional groups present on the cell walls of the bacteria or binding by bacterial extracellular polymeric substances (EPS).

Streptococcus species produces enzymes as serine proteinase, $\mathrm{N}-$ acetylglucosaminidase, cysteine proteinase, NAD-glycohydrolase, ADP-ribosyltransferase and arginine deiminase (Collin and Olsén, 
2003) and carboxylases (Arioli et al., 2009). Enzymes are well known to degrade hazardous organic compounds.

The main functional groups present on Streptoccoccus sp. surface are amide I and amide II, with absorption bands at 1653 and $1541 \mathrm{~cm}^{-1}$ respectively, phosphate bands at $1237 \mathrm{~cm}^{-1}$ and carbohydrate bands at $1070 \mathrm{~cm}^{-1}$ next to hydrocarbon absorption bands in the wavelength region between 3000 and $2800 \mathrm{~cm}^{-1}$ (Van der Mei et al., 1996). Some of these groups are responsible for the adsorption of diethylketone or its degradation intermediates and contribute to the biotreatment.

The typical degradation pathway of diethylketone was well described by Nielsen and Allard (2009) and suggests the formation of intermediates. As happened for the degradation of diethylketone using A. viscosus (Costa et al., 2012), some of these intermediates appear during the GC analysis of the aqueous solution after some hours of experiment. The presence of intermediates proves that the diethylketone is being degraded. The metabolites were identified as 2-pentanone, methyl-acetate and ethyl-acetate. These intermediates disappeared during the experiment meaning that they were also degraded by the concentrated biomass.

The presence and identification of the intermediates allowed an approach to the metabolic degradation pathway of diethylketone (DEK) by S. equisimilis. The presence of 2-pentanone only represents a change on the carbon atom where the ketone group is attached to, but the presence methyl-acetate and ethylacetate, both compounds with lower number of carbons than diethylketone, means that this last is suffering degradation. The diethylketone is degraded first as ethyl-acetate and in a second stage as methyl-acetate. As it was said, these metabolites were also consumed by the microorganism.

The removal percentages obtained with the concentrated biomass were around $100 \%$, for all the assays. The concentrated biomass allows the improvement of the biodegradation efficiency from $95 \%$ to $100 \%$. It is important to highlight that the use of diethylketone as carbon source with no addition of culture media allows a less expensive process.

\subsection{Modelling of the removal kinetics of diethylketone by S. equisimilis}

The kinetics of removal describes the rate of adsorbate uptake onto $S$. equisimilis and it controls the equilibrium time. The kinetic adsorption data were investigated to understand the dynamics of the adsorption process in terms of the order of the rate constant and this is very important for the process design and operation control of an upscale removal system. Zero order, pseudo-first order, pseudo-second order and three-half order kinetics were used to describe the removal of diethylketone by S. equisimilis. The constants for all models were evaluated and listed in Table 2 and the corresponding plots are shown as supplementary material in S1.

From Table 2, it is possible to conclude that the best fit was obtained for the pseudo-second order model with correlation coefficient around 1 . The constant $K_{2}$ decreases with the increase of the diethylketone concentration. According to Yahya et al. (2012) for processes that follow a pseudo second-order mechanism, the ratelimiting step may be chemical sorption involving valence forces through the sharing or exchange of electrons between sorbent and sorbate. In biodegradation processes usually a three-half-order kinetics model is used (Saravanan et al., 2009). This one is based on the first-order model with the introduction of an additional term to explain the biomass formation. Using this strategy, the correlation coefficient $\left(R^{2}\right)$ obtained is lower, especially for the concentration of $0.8 \mathrm{~g} \mathrm{~L}^{-1}$, than the obtained when using the pseudo-second order model.
Table 2

Constant parameters of zero-order, pseudo-first, pseudo-second and three-halforder kinetic models for different initial diethylketone concentrations.

\begin{tabular}{lllllll}
\hline \multirow{2}{*}{$S\left(\mathrm{~g} \mathrm{~L}^{-1}\right)$} & \multicolumn{2}{l}{ Zero order kinetics } & & \multicolumn{3}{l}{ Pseudo-first order kinetics } \\
\cline { 2 - 3 } \cline { 5 - 6 } & $K_{0}\left(\mathrm{~g}(\mathrm{Lh})^{-1}\right)$ & $S_{0}\left(\mathrm{~g} \mathrm{~L}^{-1}\right)$ & & $R^{2}$ & $K_{1}(\mathrm{~h})$ & $R^{2}$ \\
\hline 0.8 & 0.0350 & 0.22 & & 0.722 & 0.0059 & 0.562 \\
1.6 & 0.0424 & 0.55 & & 0.864 & 0.0313 & 0.959 \\
2.4 & 0.0345 & 0.66 & & 0.835 & 0.0256 & 0.941 \\
3.2 & 0.0348 & 1.14 & & 0.888 & 0.0234 & 0.950 \\
3.9 & 0.0523 & 1.53 & & 0.937 & 0.0366 & 0.970
\end{tabular}

$S\left(\mathrm{~g} \mathrm{~L}^{-1}\right) \quad$ Pseudo-second order kinetickree-half order kinetics

\begin{tabular}{lllllrl}
\cline { 6 - 7 } \cline { 6 - 7 } & $K_{2}\left(\mathrm{~g}(\mathrm{mg} \mathrm{h})^{-1}\right)$ & $R^{2}$ & & $K_{31}\left(1 \mathrm{~h}^{-1}\right)$ & $K_{32}\left(1 \mathrm{~h}^{-2}\right)$ & $R^{2}$ \\
\hline 0.8 & 22.7800 & 1.000 & 0.0501 & 0.0002 & 0.249 \\
1.6 & 5.6556 & 0.999 & 0.0641 & -0.0004 & 0.997 \\
2.4 & 5.9775 & 1.000 & 0.0381 & $-1.8 \mathrm{E}-4$ & 0.987 \\
3.2 & 1.8377 & 0.999 & 0.0251 & $-1.6 \mathrm{E}-4$ & 0.999 \\
3.9 & 0.6370 & 1.000 & 0.0274 & -0.0002 & 0.996 \\
\hline
\end{tabular}

\section{Conclusions}

The results of this research showed the potential of pure bacterial cultures of $S$. equisimilis for biodegradation of diethylketone. Different growth kinetic models were applied to the data. The removal percentages obtained are very promising and show that this system could be applied to the treatment of wastewater contaminated with solvents. An important approach to the metabolic pathway of diethylketone by $S$. equisimilis was developed. Diethylketone is degraded to acetate species (methyl and ethyl) probably catalyzed by carboxylases.

\section{Acknowledgements}

The authors would like to gratefully acknowledge the financial support of this project by the Fundação para a Ciência e Tecnologia, Ministério da Ciência e Tecnologia, Portugal and co-funding by FSE (programme QREN - POPH) - PEst-OE/EQB/LA0023/2013. The authors thank the Project "BioEnv-Biotechnology and Bioengineering for a sustainable world”, REF. NORTE-07-0124-FEDER-000048, co-funded by the Programa Operacional Regional do Norte (ON.2 - O Novo Norte), QREN, FEDER. Cristina Quintelas thanks Cristina Quintelas thanks FCT for a Post-Doc (SFRH/BPD/32113/2006) and Filomena Costa thanks FCT for a PhD grant (SFRH/BD/77666/2011).

\section{Appendix A. Supplementary data}

Supplementary data associated with this article can be found, in the online version, at http://dx.doi.org/10.1016/j.ecoleng. 2014.05.009.

\section{References}

Akar, T., Arslan, S., Akar, S.T., 2013. Utilization of Thamnidium elegans fungal culture in environmental cleanup: a reactive dye biosorption study. Ecol. Eng. 58, 363-370.

Andrews, J.F., 1968. A mathematical model for the continuous culture of microorganisms utilizing inhibitory substance. Biotechnol. Bioeng. 10, 707-723.

Arioli, F., Roncada, P., Salzano, A.M., Deriu, F., Corona, S., Guglielmetti, S., Bonizzi, L., Scaloni, A., Mora, D., 2009. The relevance of carbon dioxide metabolism in Streptococcus thermophilus. Microbiology 155, 1953-1965.

Collin, M., Olsén, A., 2003. Extracellular enzymes with immunomodulating activities: variations on a theme in Streptococcus pyogenes. Infect. Immun. 71, 2983-2992.

Costa, F., Quintelas, C., Tavares, T., 2012. Kinetics of biodegradation of diethylketone by Arthrobacter viscosus. Biodegradation 23, 81-92. 
Das, D., Vimala, R., Das, N., 2014. Biosorption of Zn(II) onto Pleurotus platypus: 5-level Box-Behnken design, equilibrium, kinetic and regeneration studies. Ecol. Eng. $64,136-141$.

Hasan, S.A., Wietzes, P., Janssen, D.B., 2012. Biodegradation kinetics of 4 fluorocinnamic acid by a consortium of Arthrobacter and Ralstonia strains. Biodegradation 23, 117-125.

Lin, H.-Y., Yu, C.-P., Chen, Z.-L., 2013. Aerobic and anaerobic biodegradation of TNT by newly isolated Bacillus mycoides. Ecol. Eng. 52, 270-277.

Luong, J.H.T., 1986. Generalization of Monod kinetics for analysis of growth data with substrate inhibition. Biotechnol. Bioeng. 29, 242-248.

Monod, J., 1949. The growth of bacterial cultures. Annu. Rev. Microbiol. 3, 371-394.

Nielsen, A., Allard, A.S., 2009. Chemistry of organic pollutants. In: Sabljic, A. (Ed.) Environmental and Ecological Chemistry, vol. 1. Eolss, ISBN 978-1-84826-186-0, pp. 1-424 (eBook).

Powell, E.O., 1967. The growth rate of microorganisms as function of substrate concentration. In: Evans, C.G.T., Strange, R.E., Tempest, W.(Eds.), Microbial Physiology and Continuous Culture. HMSO, London, United Kingdom.
Quintelas, C., Costa, F., Tavares, T., 2013. Bioremoval of diethylketone by the synergistic combination of microorganisms and clays: uptake, removal and kinetic studies. Environ. Sci. Pollut. Res. 20, 1374-1383.

Quintelas, C., Figueiredo, H., Tavares, T., 2011. The effect of clay treatment on remediation of diethylketone contaminated wastewater: uptake, equilibrium and kinetic studies. J. Hazard. Mater. 186, 1241-1248.

Saravanan, P., Pakshirajan, K., Saha, P., 2009. Batch growth kinetics of an indigenous mixed microbial culture utilizing $\mathrm{m}$-cresol as the sole carbon source. J. Hazard. Mater. 162, 476-481.

Tani, A., Hewitt, C.N., 2009. Uptake of aldehydes and ketones at typical indoor concentrations by houseplants. Environ. Sci. Technol. 43, 8338-8343.

Van der Mei, H.C., Naumann, D., Busscher, H.J., 1996. Grouping of Streptococcus miffs strains grown on different growth media by FT-IR. Infrared Phys. Technol. 37 , 561-564.

Yahya, S.K.,Zakaria, Z.A., Samin, J., Rajd, A.S.S., Ahmada, W.A., 2012. Isotherm kinetics of $\mathrm{Cr}(\mathrm{III})$ removal by non-viable cells of Acinetobacter haemolyticus. Colloids Surf B 94, 362-368. 